Reprinted with permission from: Environmental Entomology. August 1994.

23(4):1006-1012.

Published and copyrighted by: ${ }^{\circledR}$ Entomological Society of America. www.entsoc.org

\title{
Biology and host preference of Oxicesta geographica (Lepidoptera: Noctuidae) candidate agent for biological control of Euphorbia esula complex (Euphorbiaceae) in North America ${ }^{1}$
}

\author{
M. CRISTOFARO, A. GASSMANN, and P. PECORA \\ Current address for Cristofaro and Pecora: USDA-ARS European Biological Control Laboratory International Insti- \\ tute of Biological Control, Batiment B1, Parc Scientifique, Agropolis II Boulevard du Lironde 34980, Montferrier sur \\ Lez, France. Current Address for Gassman: USDA-ARS European Biological Control Laboratory International Insti- \\ tute of Biological Control, 1, Chemin des Grillons, 2800 Delémont, Switzerland.
}

\begin{abstract}
:
The biology and host specificity of Oxicesta geographica F. from Romania, Hungary, and Southwestern Russia were studied to evaluate the potential of this moth as a new biological control agent of leafy spurge, Euphorbia esula L., "complex" in North America. This oligophagous, multivoltine tent caterpillar is common on perennial spurges in southeastern Europe and Asia Minor and prefers dry, open sites. Results of nochoice feeding tests with first instars on 93 plant species and biotypes, distributed in 33 families, show that $O$. geographica completed its life cycle mainly on plants of Euphorbia, subgenus Esula, and occasionally fed and developed on species in other subgenera of the genus. Studies of late instars did not show any important extensions of the host range.
\end{abstract}

Keywords:

Noctuid moth, host range, larval survival test.

Leafy spurge, Euphorbia esula L. (Euphorbiaceae) (=E. virgata Waldstein \& Kitaibel of many authors, Dunn 1979), is a complex of species and biotypes of Eurasian origin that has become a serious problem in North American rangelands, pastures, and recently

\footnotetext{
${ }^{1}$ Received for publication 6 March 1992; accepted 3 January 1994.
} 
in cropland and urban areas. It becomes an irreversibly dominant weed on rangelands and pastures, displacing useful forage plants (Harris et al. 1985) and produces an irritant that causes dermatitis to humans and animals (Kingsbury 1964). The problem is most severe on uncultivated lands, but leafy spurge can also reduce crop yields by $10-100 \%$ (Derscheid \& Wrage 1972). Leafy spurge infested 451 counties in 25 states in the United States at the time of Dunn's (1979) study. The area of most serious infestation in North America is defined by a circle $1,930 \mathrm{~km}(1,200 \mathrm{mi})$ in diameter, centered in northeastern Montana; this area covers parts of nine states in the United States and five provinces in Canada and encompasses 1 million ha (nearly 2.5 million acres) (Lacey et al. 1985). A conservative estimate of losses to leafy spurge in the United States, in terms of expenditures for control and losses of rangeland productivity, was given as $\$ 10.5$ million annually by Noble et al. (1979). A biological control program against leafy spurge was started by Agriculture Canada in 1962 and by the Agricultural Research Service, U.S. Department of Agriculture in 1973, and 11 insect species have been evaluated and introduced as biological control agents in North America to date (Harris et al. 1985, Pecora \& Dunn 1990). Although six species have become established, it is likely that effective biological control will be achieved only if the combined attack of natural enemies in various climatic zones and habitat types extends over the entire vegetative season (Pecora \& Dunn 1990). This article reports our studies on the biology and larval host range of the moth Oxicesta geographica F. (Lepidoptera: Noctuidae), a specialized defoliator of Euphorbia spp. in southeastern Europe and Asia Minor.

\section{Materials and methods}

The studies were conducted at the Biological Control of Weeds Laboratory USDAARS Europe, Rome, Italy (1984 to 1990) and the CAB International Institute of Biological Control (IIBC) Laboratory, Delemont, Switzerland (1987 to 1989).

Host Plants and Geographical Distribution. Larvae of $O$. geographica have been reported to be associated with E. stepposa Zoz ex Prokhanov (Popescu-Gorj \& Draghia 1974, Popescu-Gorj \& Konig 1976) and E. cyparissias L. (Seitz 1914). Spüler (1908) and Lhomme (1923) recorded the species on common toadflax (Linaria vulgaris L. (Scrophulariaceae)), but these authors probably misidentified the host because of the similar appearance of some Euphorbia species and L. vulgaris before flowering.

Oxicesta geographica is a species of the continental steppe biome; it is found predominantly in areas of open light sandy soils and dry conditions or rangeland pastures of low productivity, the typical habitats of E. seguieriana Necker, and E. stepposa in eastern Europe, or in small undisturbed areas around cultivated fields (e.g., corn, sugar beet, wheat) on E. virgata. The establishment of the moth appears to depend on a high density of spurge population. It is known from southern Romania (Popescu-Gorj \& Konig 1976), Austria, Hungary, Yugoslavia to northern Greece, Turkey, and Russian Moldavia and Georgia (former southwestern USSR). The distribution of O. geographica is shown in Fig. 1.

Page 2 of 13 
The genus Oxicesta Hübner (1822) belongs to the family Noctuidae, subfamily Pantheinae (Poole 1989). Balachowsky (1972) listed four pest species belonging to the subfamily, all in the genus Apatele Hübner 1809-13. These species feed mainly on forest trees, but may attack fruit trees or cultivated plants in the genera Beta, Brassica, Fragaria, Humulus, Lavandula, and Medicago. Simyra dentinosa Freyer, another noctuid moth that is being studied by the ARS as a biological control agent of leafy spurge, belongs to the same subfamily.

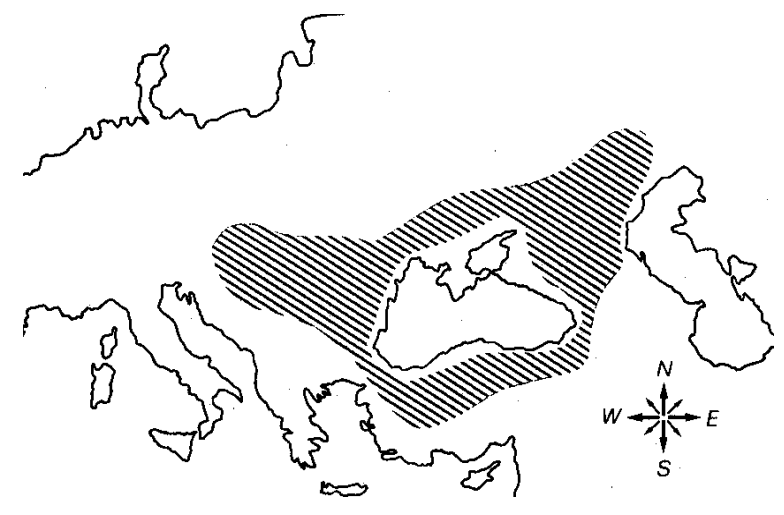

Fig. 1. Distribution of $O$. geographica in Eurasia.

The other two palaearctic species of Oxicesta have also been recorded on plants of the genus Euphorbia. Oxicesta chamaesyces Guenee was found on E. chamaesyce L. (Seitz, 1914), E. characias L. and E. stepposa (Spüler, 1908), and on Sedum sp. (Crassulaceae) (Lhomme, 1923). Garcia-Barros (1984) noted that $O$. serratae Zerny is associated with $E$. serrata L.

Host Range of Larvae. Host range was determined in laboratory tests by exposing neonate larvae to 101 species and varieties of plants, which are distributed in 33 families. Heywood (1978) was used as a guide in constructing the test plant list. Larvae used in the tests were reared from eggs collected in the field (first generation larvae), or from eggs laid in the laboratory by adults reared from larvae collected in the field (second or third generations). Two methods were used for conducting the tests.

Method A. (USDA-ARS laboratory for Romanian material). Host range was determined in two laboratory tests by exposing neonate larvae to 39 species and varieties of plants, which are distributed in 13 families. Five neonate larvae were placed in each cage (transparent plastic tubes; $20-\mathrm{cm}$ diameter, $50-\mathrm{cm}$ height), which enclosed a potted plant (greatest diameter $22 \mathrm{~cm}$ ). There were five plants per species, and controls were E. virgata plants from the collection site in eastern Romania. Fresh plants were replaced when necessary. Tests were conducted under natural photoperiod, $23.5 \pm 6.8^{\circ} \mathrm{C}$, and $61 \pm 20 \%$ RH, between 12 May and 13 July 1988.

During 1990 an additional larval survival test was carried out with 22 plant species of which 15 are in the family Euphorbiaceae (14 in the genus Euphorbia), and seven are in other families. Two O. geographica populations (from Romania and Southwestern Russia) were used for these tests. A minimum of five replications were made for each plant, using ten neonate larvae on each potted plant. Three replications were made using neonate larvae from eggs collected near Focsani (Romania), and two with first instars from Stavropol Southwestern Russia. Additional replications were made testing seventy third instars (for target test plants) from the two populations to determine if the feeding behavior is different during the development of the larvae. 
Method B. (IIBC laboratory for Russian and Hungarian material). During 1987, tests were made with material from the Southwestern Russia and Hungarian populations, and their progeny. During 1988 and 1989, tests were made exclusively with a Hungarian population. Colonies of first to third instars, found at Tompa, southern Hungary on $E$. stepposa and E. seguierana, were reared on Canadian leafy spurge until pupation under laboratory conditions. All of the pupae were kept in the laboratory until adult emergence. Adults were reared in 1-liter plastic cylinders with the walls covered with white paper. Eggs, laid on the paper, were incubated at $20 \pm 1^{\circ} \mathrm{C}$, and a photoperiod of 16:8 (L:D) hours. Neonate larvae were transferred in groups of 10 and 20 to petri dishes $9-14-\mathrm{cm}$ diameter and provided with leaves and cut shoots of the test plant species. Larger larvae were reared in half-liter plastic containers. No-choice larval feeding tests were conducted from July until mid-September (temperature $20 \pm 1^{\circ} \mathrm{C}$, photoperiod 16 hours). A minimum of 20 first instars was used for each of the 44 plant species tested. The potential extension of the host range by late instars, previously reared on a Canadian biotype of leafy spurge or E. lucida Waldestein \& Kitaibel, was studied using groups of 10-20 thirdinstars from the Hungarian population for each of the 20 plant species tested.

Data Analysis. The data were analyzed by using the procedures of the Michigan State University Microcomputer Statistical Program (MSTAT 1986). Regression analysis was used to examine the relationship between temperature and insect development. The variability of the differences in larval survival rate and in the time spent to complete larval development was analyzed with analysis of variance (ANOVA) and with the Duncan's (1955) multiple-range test. The remaining survival data were analyzed with $t$-test, to determine if larval development was dependent on the plant species, larval instars, and replication effects.

\section{Results}

Life History. Adults apparently emerge in nature during late April to early May, as eggs were found as early as 2 May, 1989 in Romania. Young colonies (first and second instars) were found during May to early June in Hungary and Romania; mature larvae were collected during late October in Romania.

Oxicesta geographica has five instars. There were four generations in the laboratory. Because, in nature, eggs were collected on 2 May and 10 June, the first three instars were collected on 10 June, and mature larvae were collected on 20 October, it can be assumed that the species breeds continuously during the warm part of the year. Larvae fed in silken webs on the apices of Euphorbia spp. plants, causing extensive damage, First instars, typically in groups of 20-30, fed on flower buds and tender leaves for $\approx 4$ days before they molted and moved together to a new branch or group of branches or to a new plant. Fifth instars were solitary feeders and consumed less than the other instars.

In the laboratory, larvae reached the pupal stage after $\approx 4 \mathrm{wk}\left(26.8 \pm 5.3^{\circ} \mathrm{C}\right.$, and 46.5 $\pm 16.5 \mathrm{RH})$; they pupated in light yellow silk cocoons spun on stems. The pupal stage lasted 9 to 13 days. 
Fifteen pairs of adults lived 5 to 8 days under laboratory conditions. Oviposition started 1 to 3 days after mating. Eggs were laid mostly on the lower surfaces of leaves of Euphorbia. The number of eggs laid per female ranged between 60 and about 450. In nature, single egg batches consist of $>300$ eggs. The egg stage lasted 9 to 12 days and egg fertility was $99 \%$.

The rate of egg, larval, and pupal development is correlated with temperature $(\mathrm{P}<$ 0.05 ) (Table 1). The incubation period was 6 days at $30^{\circ} \mathrm{C}$ but 30 days at $15^{\circ} \mathrm{C}$. The percentage of hatching at these temperatures was also variable, probably a result of inadequate humidity. No eggs hatched at $10^{\circ} \mathrm{C}$.

Table 1. Effects of temperature on development rate (for $50 \%$ of the population) and survival of immature stages of $O$. geographica

\begin{tabular}{|c|c|c|c|c|c|c|c|c|c|c|c|}
\hline \multirow[b]{3}{*}{ Stage } & \multicolumn{10}{|c|}{ Temperature, ${ }^{\circ} \mathrm{C}$} & \multirow[b]{3}{*}{$r^{2}$} \\
\hline & \multicolumn{2}{|c|}{10} & \multicolumn{2}{|c|}{15} & \multicolumn{2}{|c|}{20} & \multicolumn{2}{|c|}{25} & \multicolumn{2}{|c|}{30} & \\
\hline & $\begin{array}{l}\text { No. } \\
\text { days }\end{array}$ & $\begin{array}{c}\% \\
\text { Surv. }\end{array}$ & $\begin{array}{l}\text { No. } \\
\text { days }\end{array}$ & $\begin{array}{c}\% \\
\text { Surv. }\end{array}$ & $\begin{array}{l}\text { No. } \\
\text { days }\end{array}$ & $\begin{array}{c}\% \\
\text { Surv. }\end{array}$ & $\begin{array}{l}\text { No. } \\
\text { days }\end{array}$ & $\begin{array}{c}\% \\
\text { Surv. }\end{array}$ & $\begin{array}{l}\text { No. } \\
\text { days }\end{array}$ & $\begin{array}{c}\% \\
\text { Surv. }\end{array}$ & \\
\hline Egg development & - & 0.0 & 30 & 20.0 & 13 & 50.0 & 8.5 & 50.0 & 6 & 5.0 & 0.999 \\
\hline First instar & 22 & 0.0 & 13 & 90.0 & 7 & 93.8 & 4.5 & 97.5 & 3 & 92.5 & 0.961 \\
\hline Second instar & - & - & 12 & 72.5 & 6 & 86.3 & 4.5 & 87.5 & 2.5 & 87.5 & 0.938 \\
\hline Third instar & - & - & 11 & 35.0 & 7 & 73.8 & 5.0 & 77.5 & 3 & 82.5 & 0.942 \\
\hline Fourth instar & - & - & 20 & 0.0 & 5 & 62.5 & 4.0 & 75.5 & 2 & 82.5 & 0.966 \\
\hline Fifth instar & - & - & 0 & 0.0 & 19 & 20.0 & 14 & 40.0 & 8 & 67.5 & 0.923 \\
\hline $\begin{array}{l}\text { Pupal } \\
\text { development }\end{array}$ & - & 0.0 & 34 & 40.0 & 20 & 73.3 & 10 & 44.0 & 8.5 & 70.0 & 0.964 \\
\hline Total $^{\mathrm{a}}$ & - & - & - & - & 77 & 2.8 & 50.5 & 4.4 & 33 & 1.3 & - \\
\hline
\end{tabular}

Larvae did not develop at $10^{\circ} \mathrm{C}$, but a few first instars survived for $3 \mathrm{wk}$ at this temperature. About $35 \%$ of the larvae reached the fourth instar at $15^{\circ} \mathrm{C}$, however, these larvae were very small and all died within 20 days. At $20^{\circ} \mathrm{C}$ only $20 \%$ of the larvae completed their development after 44 days and moths emerged within 20 days after pupation. No continuous development was possible at 10 and $15^{\circ} \mathrm{C}$.

In summary, the period of time required for one generation (egg to adult) ranged from 33 days at $30^{\circ} \mathrm{C}$ to 77 days at $20^{\circ} \mathrm{C}$, but development at the higher temperature was accompanied by very high egg mortality.

Two parasitoids, Aleiodes rugulosus (Nees) and Apanteles sp. (both Hymenoptera: Braconidae), were reared from the few larvae collected in Romania during October 1984 and 1988, respectively; the percentage of parasitism (only Apanteles sp.) of the Hungarian population was very low. 
Host Range Tests with First Instars. Larvae completed development on 13 species in the genus Euphorbia and on four biotypes of North American leafy spurge (Tables 2, 3 , and 4).

Table 2. Plant species or varieties used in no-choice feeding tests with first instars of o. geographica.

\begin{tabular}{|c|c|c|c|}
\hline & Plants related & spurge (Euphorbiaceae) & \\
\hline Order & Subgenus & Species & \\
\hline Euphorbiales & Esula & Euphorbia virgata W. \& K.-Focsania & a \\
\hline & & E. virgata-Saskatchewan ${ }^{\mathrm{a}}$ & $\mathrm{bc}$ \\
\hline & & E. virgata-Nebraska ${ }^{\mathrm{a}}$ & a \\
\hline & & E. virgata-Wisconsin ${ }^{\mathrm{a}}$ & $\mathrm{a}$ \\
\hline & & E. virgata-Montana ${ }^{\mathrm{a}}$ & $\mathrm{a}$ \\
\hline & & E. ceratocarpa Tenore ${ }^{\mathrm{a}}$ & $\mathrm{a}$ \\
\hline & & E. oblongata Grisebach ${ }^{\mathrm{a}}$ & $\mathrm{c}$ \\
\hline & & E. palustris $\mathrm{L}^{\mathrm{a}}$ & $\mathrm{b}$ \\
\hline & & E. polychroma Kerner von Marilau & $\mathrm{c}$ \\
\hline & & E. cyparissias $\mathrm{L}^{\mathrm{a}}$ & $\mathrm{b}$ \\
\hline & & E. lucida W. \& K. ${ }^{\mathrm{a}}$ & $\mathrm{bc}$ \\
\hline & & E. seguieriana Necker $^{a}$ & b \\
\hline & & E. amygdaloides $\mathrm{L}^{\mathrm{a}}$ & $\mathrm{c}$ \\
\hline & & E. stepposa Zoz ex Prokhanov ${ }^{\mathrm{a}}$ & b \\
\hline & & E. puplus L. & b \\
\hline & & E. dendroides L. & a \\
\hline & & E. characias L. & a \\
\hline & & E. lathyris L. & $a b$ \\
\hline & & E. platyphyllos $\mathrm{L}$. & b \\
\hline & & E. roemerana Scheele & a \\
\hline & & E. wulfenii (Hoppe ex Koch) & $\mathrm{c}$ \\
\hline & Agaloma & E. corollata $\mathrm{L}$. & $a b$ \\
\hline & & E. marginata Pursh & $a b$ \\
\hline & & E. antisyphilitica Zuccar & $a b$ \\
\hline & Myrsinitae & E. myrsinites L. ${ }^{\mathrm{a}}$ & $\mathrm{c}$ \\
\hline & & E. rigida Bieb. & a \\
\hline & Euphorbium & E. tirucalli $\mathrm{L}$. & $\mathrm{ac}$ \\
\hline & & E. milii Desmoulins & $\mathrm{a}$ \\
\hline & & E. milii Desmoulins ${ }^{\mathrm{a}}$ & $b-c$ \\
\hline & Chamaesyce & E. maculata $\mathrm{L}^{\mathrm{a}}$ & $a b$ \\
\hline & & E. supina Rafinesque-Schmaltz & $\mathrm{a}$ \\
\hline & & E. serpyllifolia Persoon & $\mathrm{a}$ \\
\hline & Poinsettia & E. heterophylla L. & a \\
\hline & & E. pulcherrima Willdenow & $\mathrm{ac}$ \\
\hline & & Acalypha hispida N. L. Burman & $\mathrm{c}$ \\
\hline & & Codieaum variegatum Blume & $a b$ \\
\hline & & Mercurialis anпиа $\mathrm{L}$. & $\mathrm{a}$ \\
\hline & & Mercurialis perennis L. & $\mathrm{c}$ \\
\hline & & Pedilanthus tithymaloides $\mathrm{L}$. & $\mathrm{c}$ \\
\hline & & Pedilanthus macrocarpus Bentham & $\mathrm{b}$ \\
\hline & & Manihot esculenta Crantz & b \\
\hline & & Ricinus communis $\mathrm{L}$. & $a b$ \\
\hline
\end{tabular}




\begin{tabular}{|c|c|c|c|}
\hline \multicolumn{3}{|c|}{ Plants related to leafy spurge (Euphorbiaceae) } & \\
\hline Order & Subgenus & \multicolumn{2}{|l|}{ Species } \\
\hline \multirow[t]{2}{*}{ Violales } & Cistaceae & Helianthemum apermium L. & $\mathrm{a}$ \\
\hline & & H. nummularium (L.) Miller & $\mathrm{b}$ \\
\hline \multirow[t]{5}{*}{ Geraniales } & Geraniaceae & Geranium rotundifolium L. & $\mathrm{a}$ \\
\hline & & Pelargonium zonale Aiton & $\mathrm{a}$ \\
\hline & & Petargonium sp. & $\mathrm{b}$ \\
\hline & Linaceae & Linum usitatissimum L. & ac \\
\hline & & Linum flavum L. & $\mathrm{b}$ \\
\hline \multirow[t]{6}{*}{ Asterales } & Compositae & Cynara scolymus L. & $\mathrm{a}$ \\
\hline & & Lactuca sativa L. & $\mathrm{a}$ \\
\hline & & Achillea millefolium L. & $\mathrm{b}$ \\
\hline & & Artemisia absinthium L. & $\mathrm{b}$ \\
\hline & & Centaurea maculosa Gussone & $\mathrm{b}$ \\
\hline & & Parthenium argentatum Gray & $\mathrm{b}$ \\
\hline Fabales & Leguminosae & Medicago sativa L. & $\mathrm{a}$ \\
\hline \multirow[t]{3}{*}{ Fagales } & Corylaceae & Carpinus betulus L. & $\mathrm{b}$ \\
\hline & Fagaceae & Quercus sp. & $\mathrm{b}$ \\
\hline & Betulaceae & Betula sp. & $\mathrm{b}$ \\
\hline Salicales & Salicaceae & Populus sp. & $\mathrm{b}$ \\
\hline \multirow[t]{2}{*}{ Sapindales } & Ruataceae & Ruta graveolens L. & $\mathrm{a}$ \\
\hline & Hippocastanaceae & Aesculus hippocastanum L. & $\mathrm{b}$ \\
\hline Ericales & Ericaceae & Calluna vulgaris Hull & $\mathrm{b}$ \\
\hline \multirow[t]{2}{*}{ Scrophuraliales } & Scrophulariaceae & Linaria vulgaris Miller & $\mathrm{ab}$ \\
\hline & & Verbascum lycnitis L. & $\mathrm{b}$ \\
\hline \multirow[t]{5}{*}{ Polemoniales } & Convolvulaceae & Ipomoea grandiflora Roxburg & $\mathrm{a}$ \\
\hline & & Ipomoea batatas L. & $\mathrm{b}$ \\
\hline & Solanaceae & Solanum tuberosum L. & $\mathrm{b}$ \\
\hline & & Lavandula angustifolia Miller & $\mathrm{b}$ \\
\hline & Labiatae & Mentha spicata L. & $\mathrm{b}$ \\
\hline \multirow[t]{2}{*}{ Polygonales } & Polygonaceae & Rumex acetosa L. & $\mathrm{b}$ \\
\hline & & Rheum raponticum $\mathrm{L}$. & $\mathrm{b}$ \\
\hline \multirow[t]{3}{*}{ Capparales } & Cruciferae & Alyssum argentum Allioni & $\mathrm{b}$ \\
\hline & & Brassica oleracea L. & $\mathrm{b}$ \\
\hline & & Brassica sp. & $\mathrm{a}$ \\
\hline \multirow[t]{6}{*}{ Gentianales } & Asclepiadaceae & Asclepias syriaca L. & $a b$ \\
\hline & & Asclepias speciosa Torry & $\mathrm{a}$ \\
\hline & & Vincetoxycum hirundunaria Medicus & $\mathrm{b}$ \\
\hline & & Hoya bella W. J. Hooker & $\mathrm{b}$ \\
\hline & Apocynaceae & Vinca major L. & $\mathrm{a}$ \\
\hline & Umbelliferae & Daucus carota L. & $\mathrm{b}$ \\
\hline \multirow[t]{7}{*}{ Rosales } & Rosaceae & Rosa sp. & $\mathrm{a}$ \\
\hline & & Sanguisorba minor Scopoli & $\mathrm{b}$ \\
\hline & & Fragaria vesca $\mathrm{L}$. & $\mathrm{a}$ \\
\hline & & Malus sp. & $\mathrm{b}$ \\
\hline & & Prunus sp. & $\mathrm{b}$ \\
\hline & Crassulaceae & Sedum album L. & $a b$ \\
\hline & & Sempervicum tectorum $\mathrm{L}$. & $\mathrm{b}$ \\
\hline Urticales & Urticaceac & Ficus elastica Roxburg & $\mathrm{a}$ \\
\hline \multirow[t]{2}{*}{ Commelinales } & Graminaceae & Triticum aestivum $\mathrm{L}$. & $\mathrm{a}$ \\
\hline & & Zea mays L. & $\mathrm{a}$ \\
\hline
\end{tabular}

Page 7 of 13 
Table 3. Larval survival test during 1990: percentage of larvae surviving to pupal stage (Romanian and Russian populations).

\begin{tabular}{|c|c|c|c|c|}
\hline \multicolumn{5}{|c|}{ Plants related to leafy spurge (Euphorbiaceae) } \\
\hline \multirow[b]{2}{*}{ Test plants } & \multicolumn{2}{|c|}{ First instars ${ }^{\mathrm{a}}$} & \multicolumn{2}{|c|}{ Third-instars $^{\mathrm{b}}$} \\
\hline & Romania & Russia & Romania & Russia \\
\hline E. virgata (control) ${ }^{\mathrm{a}}$ & 30.00 & 30.00 & 60.00 & 52.22 \\
\hline E. virgata $(\text { Oregon })^{\mathrm{a}}$ & 26.67 & 35.00 & 55.00 & 60.00 \\
\hline E. maculata (Montana) ${ }^{\mathrm{a}}$ & 0.00 & 0.00 & 13.33 & 7.50 \\
\hline E. milii & - & - & - & - \\
\hline E. corollata & - & - & - & - \\
\hline E. roemerana & - & - & - & - \\
\hline E. rigida & - & - & - & - \\
\hline E. tirucalli & - & - & - & - \\
\hline E. lathyris & - & - & - & - \\
\hline E. antisiphylitica & - & - & - & - \\
\hline E. marginata & - & - & - & - \\
\hline E. heterophylla & - & - & - & - \\
\hline E. pulcherrima & - & - & - & - \\
\hline E. supina & - & - & - & - \\
\hline Ricinus communis & - & - & - & - \\
\hline \multicolumn{5}{|c|}{ Plant species in other orders of the superorder Rosidae } \\
\hline Fragaria vesca & - & - & - & - \\
\hline Vinca major & - & - & - & - \\
\hline Rosa sp. & - & - & - & - \\
\hline Sedum album & - & - & - & - \\
\hline Humulus lupulus & - & - & - & - \\
\hline Brassica sp. & - & - & - & - \\
\hline Medicago sativa & - & - & - & - \\
\hline \multicolumn{5}{|c|}{$\begin{array}{l}\text { a, Groups of larvae on potted plants; } 10 \text { larvae on each potted plant and } 5 \text { potted plants per test plant; b, groups of } 70 \\
\text { larvae ( } 30 \text { Romanian and } 40 \text { from Russia) distributed in four potted plants. } \\
\text { a plant species on which some development of larvae occurred. }\end{array}$} \\
\hline \multicolumn{5}{|c|}{$\begin{array}{l}\text { Among the } 13 \text { plant species suitable for larval development, } 11 \text { belong to the subge- } \\
\text { nus Esula. Contrasting results (possibly caused by different rearing procedures used in } \\
\text { the two laboratories) were obtained on the two spurge species outside the subgenus } \\
\text { Esula. Euphorbia maculata (subgenus Chamaesyce), a native spurge from North Amer- } \\
\text { ica, was occasionally accepted by larvae of the Romanian population but not by those of } \\
\text { the Russian and Hungarian populations. In contrast, E. milii Desmoulins (subgenus Eu- } \\
\text { phorbium) was accepted, one time each, by the latter two, but never by the Romanian } \\
\text { population. }\end{array}$} \\
\hline
\end{tabular}


The Romanian population required less time to complete larval development $(F=$ 286.16, $\mathrm{df}=50, P<0.001)$ and the general survival rate was higher $(F=9.39$, df $=60$, $P<0.001$ ) (Table 4 ), which was possibly the result of more suitable rearing conditions (higher temperature in laboratory facilities) and better food quality (potted plants versus cut shoots and leaves). No sustained feeding was observed outside the genus Euphorbia and the family Euphorbiaceae. Survival of second and third instars was significantly lower on Carpinus betulus L. than on leafy spurge (ANOVA: $F=719.93$, $\mathrm{df}=8, P<$ 0.001 ). The second molt on $C$. betulus occurred on average four days later than on $E u$ phorbia spp., and the third instar did not gain weight and died shortly after molting.

Table 4. Larval survival test for first instars of $\boldsymbol{O}$. geographica.

\begin{tabular}{|c|c|c|c|c|c|c|c|c|}
\hline \multirow[b]{2}{*}{ Test plants ${ }^{\mathrm{a}}$} & \multirow{2}{*}{$\begin{array}{c}\text { No. } \\
\text { larvae }^{b}\end{array}$} & \multicolumn{5}{|c|}{$\%$ Larvae surviving to stage ${ }^{c}$} & \multicolumn{2}{|c|}{ No. days to reach pupal stage ${ }^{\mathrm{d}}$} \\
\hline & & II & III & IV & V & $\mathrm{P}$ & Mean $\pm \mathrm{SD}$ & $n$ \\
\hline E. virg. (control) ${ }^{\mathrm{e}}$ & $25 \mathrm{a}$ & 100 & 100 & 92 & 80.0 & $60.0 \mathrm{AB}$ & $19.87 \pm 1.51 \mathrm{~A}$ & 15 \\
\hline E. virg. (Wisconsin) & $25 \mathrm{a}$ & 100 & 80 & 72 & 60.0 & $60.0 \mathrm{AB}$ & $19.60 \pm 1.50 \mathrm{~A}$ & 15 \\
\hline E. virg. (Montana) & $25 \mathrm{a}$ & 100 & 88 & 76 & 64.0 & $60.0 \mathrm{AB}$ & $20.33 \pm 1.11 \mathrm{~A}$ & 15 \\
\hline E. virg. (Nebraska) & $25 \mathrm{a}$ & 92 & 76 & 64 & 64.0 & $64.0 \mathrm{~A}$ & $20.06 \pm 1.57 \mathrm{~A}$ & 16 \\
\hline E. virg. (Saskatchewan) & $130 \mathrm{bc}$ & 63.8 & 55.4 & 49.2 & 33.8 & $20.0 \mathrm{CD}$ & $41.25 \pm 1.60 \mathrm{~B}$ & 26 \\
\hline E. cyparissias & $120 \mathrm{~b}$ & 57.5 & 42.5 & 32.5 & 30.0 & $10.8 \mathrm{CD}$ & $44.61 \pm 2.90 \mathrm{BC}$ & 13 \\
\hline E. lucida & $110 \mathrm{bc}$ & 71.8 & 63.4 & 59.1 & 49.1 & $37.3 \mathrm{ABC}$ & $43.00 \pm 3.70 \mathrm{~B}$ & 41 \\
\hline E. seguieriana & $60 \mathrm{~b}$ & 66.7 & 60.0 & 53.3 & 35.0 & 16.7CD & $44.30 \pm 2.80 \mathrm{BC}$ & 10 \\
\hline E. oblongata & $20 c$ & 60.0 & 60.0 & 60.0 & 60.0 & 35.0ABC & $47.00 \pm 4.00 \mathrm{CD}$ & 7 \\
\hline E. amygdaloides & $20 c$ & 55.0 & 50.0 & 15.0 & 10.0 & $10.0 \mathrm{D}$ & $49.00 \pm 4.24 \mathrm{D}$ & 2 \\
\hline E. palustris & $30 b$ & 86.7 & 66.7 & 60.0 & 50.0 & $30.0 \mathrm{BC}$ & $43.00 \pm 2.29 \mathrm{D}$ & 9 \\
\hline E. polychroma & $20 c$ & 65.0 & 65.0 & 65.0 & 60.0 & $30.0 \mathrm{BC}$ & $44.50 \pm 2.43 \mathrm{~B}$ & 6 \\
\hline E. milii & $50 \mathrm{bc}$ & 4.0 & 2.0 & 2.0 & 2.0 & 2.0 & 58.0 & 1 \\
\hline E. myrsinites & $40 c$ & 20.0 & 15.0 & 12.5 & 12.5 & $7.5 \mathrm{D}$ & $53.33 \pm 2.89 \mathrm{BC}$ & 3 \\
\hline E. ceratocarpa & $25 \mathrm{a}$ & 84.0 & 64.0 & 60.0 & 40.0 & $24.0 \mathrm{CD}$ & $20.33 \pm 1.37 \mathrm{E}$ & 6 \\
\hline E. maculata & $25 \mathrm{a}$ & 84.0 & 52.0 & 24.0 & 16.0 & $8.0 \mathrm{D}$ & $21.50 \pm 0.71 \mathrm{~A}$ & 2 \\
\hline E. stepposa & $20 b$ & 90.0 & 70.0 & 75.0 & 60.0 & $20.0 \mathrm{CD}$ & $46.50 \pm 1.29 \mathrm{C}$ & 4 \\
\hline E. corollata & $20 b$ & 100 & 80.0 & - & - & - & - & \\
\hline E. peplus & $20 b$ & 70.0 & 60.0 & 45.0 & - & - & - & \\
\hline E. wulfenii & $10 \mathrm{c}$ & 10.0 & - & - & - & - & - & \\
\hline E. lathyris & $50 \mathrm{~b}$ & 40.0 & 20.0 & - & - & - & - & \\
\hline Carpinus betulus & $40 \mathrm{bc}$ & 45.0 & 20.0 & - & - & - & - & \\
\hline
\end{tabular}

${ }^{\mathrm{a}}$ Test plants on which development did not occur are reported in Table 2.

${ }^{\mathrm{b}}$ A, Romanian population; B, Hungarian population, C, Russian population.

${ }^{\mathrm{c}}$ Percentages followed by different letters are different at $\mathrm{P}=0.01$ (Duncan's multiple-range test; LSD value $=18.08$; $\mathrm{SEM}=4.805$.

${ }^{\mathrm{d}}$ Averages followed by different letters are different at $\mathrm{P}=0.01$ (Duncan's multiple-range test; LSD value $=2.914$; $\mathrm{SEM}=0.7694$ ).

${ }^{\mathrm{e}}$ E. virg. = Euphorbia virgata. 
Host Range Tests with Third Instars. The potential extension of the host range by the late instars: was tested with third instars (Hungarian population) on 36 species in 14 families (Table 5). The host range was extended to E. lathyris (subgenus Esula) with only $3 \%$ of the larvae completing their development, but no larvae reached the pupal stage on E. milii and E. maculata. Development to pupae on leafy spurge, E. cyparissias, and E. lucida, starting with third instars was significantly higher than when starting with first instars (ANOVA: $\mathrm{F}=7.89,20.25$, and 10.55, respectively, $\mathrm{df}=4, \mathrm{P}<0.05$ ). Larval development was higher on E. heterophylla L. and E. wulfenii Hoppe ex Koch, but no larvae reached the pupal stage on these hosts. Some $70 \%$ of the third instars molted to the fourth instar on E. antisyphilitica Zuccar without feeding. Some nibbling, but no larval development occurred on Carpinus betulus, confirming that it is not a suitable host plant.

During 1990, no differences were observed between Romanian and Russian populations, under laboratory conditions (Table 3). Feeding and larval development were observed only on the control (E. virgata from Romania), E. esula from Oregon, and $E$. maculata. Some differences in survival were noted between neonate and third instars on E. maculata. The percentage of larval survival was very low for neonate larvae and only one larva reached the pupal stage $(2 \%)$, but it did not complete its life cycle, compared with the third instars, seven of which reached the pupal stage (four from the Romanian population and three from the Russian population [10\%]). Two larvae completed development (one from each population [2.85\%]). No feeding was observed on any of the other plant species tested, and larvae died in 3-4 d; the lack of survival on E. milii confirmed that this species is an unsuitable host.

\section{Discussion}

O. geographica is a well-defined species, taxonomically, The host range of the larvae is restricted to the genus Euphorbia (13 species), with a clear preference for the subgenus Esula (10 species); but larvae also developed on one species in the subgenus Myrsinitae (E. myrsinites L.), one species in the subgenus Euphorbium (E. milii), and one species in the subgenus Chamaesyce (E. maculata). No feeding occurred on any of the other 80 test plant species (29 Euphorbiaceae species and varieties, and 51 species in other families). Within the subgenus Esula, only a few herbaceous species and the woody species E. dendroides, E. characias, and E. wulfenii seemed to be unsuitable host plants. The inconsistency of the larvae, among the three populations, in feeding and developing on E. corollata, E. milii, and E. maculata may have been caused by different rearing conditions or genetic differences. O. geographica is generally restricted to species in the subgenus Esula, and the larvae may feed on plants outside the subgenus Esula under certain circumstances. Although E. maculata (subgenus Chamaesyce), a native American species sympatric with leafy spurge (Pemberton 1985), proved suitable occasionally for O. geographica under no-choice conditions, this does not necessarily indicate that this plant species would be acceptable under natural conditions.

O. geographica is a continental species occurring in dry areas and has been recorded from most of the herbaceous perennial spurges present in such areas (e.g., E. seguieriana, E. stepposa, E. cyparissias, and E. virgata). In contrast, the moth has never been found on spurges growing in humid or shaded habitats (e.g., E. lucida, E. palustris, E. amygda- 
loides, or E. polychroma), although these species have demonstrated in the laboratory to be suitable host plants for development of the larvae. In addition, O. geographica has never been recorded on any annual species.

Oviposition preferences and larval host ranges for insects that feed externally are not always correlated (Wiklund 1975), and it may be that the adults of O. geographica will not oviposit on many species that are suitable for larval development. Additional multiple-choice host-specificity tests, and open-field oviposition tests with critical U.S. biotypes of Euphorbia spp. should be conducted to gain a better understanding of this insect as a biological control agent.

Table 5. Larval development of third instars of $O$. geographica (Hungarian population) and survival to stages $I V, V$, and $P$.

\begin{tabular}{|c|c|c|c|c|}
\hline \multirow[b]{2}{*}{ Plant species } & \multirow[b]{2}{*}{ No. of larvae } & \multicolumn{3}{|c|}{$\%$ Larvae surviving to stage } \\
\hline & & IV & $\mathrm{V}$ & $\bar{P}$ \\
\hline Euphorbia virgata $^{\mathrm{a}, \mathrm{b}}$ & 30 & 93.3 & 63.3 & 40.0 \\
\hline E. cyparissias $^{\mathrm{a}}$ & 10 & 100 & 100 & 60.0 \\
\hline E. lucida $^{\mathrm{a}}$ & 30 & 93.3 & 76.7 & 56.7 \\
\hline E. lathyris ${ }^{\mathrm{a}}$ & 30 & 66.7 & 33.3 & 3.3 \\
\hline E. myrsinites ${ }^{\mathrm{a}}$ & 10 & 100 & 100 & 30.0 \\
\hline E. wulfenii & 10 & 90.0 & 40.0 & - \\
\hline E. milii & 20 & 66.7 & 53.3 & - \\
\hline E. heterophylla & 20 & - & - & - \\
\hline E. marginata & 20 & - & - & - \\
\hline E. pulcherrima & 20 & - & - & - \\
\hline E. tirucalli & 20 & - & - & - \\
\hline Acalypha hispida & 10 & - & - & - \\
\hline Mercurialis perennis & 10 & - & - & - \\
\hline Ricinus communis & 20 & - & - & - \\
\hline Pedilanthus tithymaloides & 10 & - & - & - \\
\hline Manihot esculenta & 20 & - & - & - \\
\hline Calluna vulgaris & 20 & - & - & - \\
\hline Solanum tuberosum & 20 & - & - & - \\
\hline Rumex acetosa & 20 & - & - & - \\
\hline Sanguisorba minor & 20 & - & - & - \\
\hline Betula sp. & 20 & - & - & - \\
\hline Malus sp. & 10 & - & - & - \\
\hline Rosa sp. & 20 & - & - & - \\
\hline Carpinus betulus & 20 & - & - & - \\
\hline Corylus sp. & 10 & - & - & - \\
\hline Medicago sativa & 10 & - & - & - \\
\hline Populus sp. & 10 & - & - & - \\
\hline Asclepias syriaca & 30 & - & - & - \\
\hline Vincetoxycum hirundinaria & 10 & - & - & - \\
\hline Daucus carota & 20 & - & - & - \\
\hline Brassica oleracea & 20 & - & - & - \\
\hline Achillea millefolium & 10 & - & - & - \\
\hline Parthenium argentatum & 20 & - & - & - \\
\hline Humulus lupulus & 10 & - & - & - \\
\hline
\end{tabular}




\section{Acknowledgments}

Special thanks are due to J.H. Williamson (First Secretary at the U.S. Embassy in Bucharest) and A. Pavel (Agriculture Section of the same Embassy) for their information and support during the survey trips in Romania; O. Kovalev (Academy of Sciences, St. Petersburg, Russia) for insect collection in the former U.S.S.R.; P.M. Marsh (USDAARS, Systematic Entomology Laboratory (SEL), Beltsville, MD) for identification of parasitoids; R. W. Poole (SEL) and L. Ronkay, Natural History Museum, Budapest) for identification of O. geographica; and to S.L. Clement (ARS, Pullman, WA); E. GarciaBarros (Department of Zoology, University of Madrid, Spain); L. Knutson (USDA-ARS European Biological Control Laboratory); R.W. Pemberton (USDA-ARS, Seoul, Korea); and D. Schroeder, (IIBC Delemont Laboratory), for reviewing the manuscript. Special thanks also go to A.C. Pastorino, M. Stazi, and the technical staff at both the Delémont Laboratory and the Rome Laboratory.

\section{References cited}

Balachowsky, A. S. 1972. Entomologie appliquée à l'agriculture. Masson et Cie Editeurs, Paris. 2:1255-1520.

Derscheid, L. A. \& L. J. Wrage. 1972. Leafy spurge. S. Dakota State Univ. Ext. For. Serv. 449.

Duncan, D. B. 1955. Multiple range and multiple F tests. Biometrics 11:1-42.

Dunn, P. H. 1979. The distribution of leafy spurge (Euphorbia esula) and other weedy Euphorbia spp. in the United States. Weed Sci. 27: 509-516.

Garcia-Barros, E. 1984. Morfologia de las fasas preimaginales y observaciones sobre la biologia de Oxicesta serratae Zerny, 1927 (Lep., Noctuidae). Bol. Asoc. Esp. Entomol. 8:111-120.

Harris, P., P. H. Dunn, D. Schroeder \& R. Vonmoos. 1985. Biological control of leafy spurge in North America. Monogr. Ser. Weed Sci. Soc. Am. 3: 79-92.

Heywood, V. H. 1978. Flowering plants of the world. Mayflower Books, NY.

Kingsbury, J. M. 1964. Poisonous plants of the United States and Canada. Prentice Hall, Englewood Cliffs, NJ.

Lacey, C. A., P. K. Fay, R. G. Lym, C. G. Messersmith \& H. P. Alley. 1985. The distribution, biology and control of leafy spurge. Mont. State Univ. Coop. Ext. Serv. Circ. 309.

Lhomme, L. [ed.]. 1923. Catalogue des Lepidopteres de France et de Belgique. 1:1-208.

MSTAT. 1986. Microcomputer statistical program: user's guide. Michigan State University, East Lansing.

Noble, D., P. H. Dunn \& L. A. Andres. 1979. The leafy spurge problem. Proceedings, Leafy Spurge Symposium. N. Dakota State Univ. Coop. Ext. Serv. Bismarck.

Pecora, P. \& P. H. Dunn. 1990. Insect associations on leafy spurge in Europe: implication for strategies for release of biological control agents in North America, pp. 75-82. In E. S. Delfosse [ed.], Proceedings, Seventh International Symposium on Biological Control of Weeds, Rome, Italy.

Pemberton, R. W. 1985. Native plant considerations in the biological control of leafy spurge. pp. 365-90. In E. S. Delfosse [ed.], Proceedings, Sixth International Symposium on Biological Control of Weeds, Vancouver, Canada.

Poole, R. W. 1989. Lepidopterorum Catalogus. 2: Fasc. 118. Brill Publishing, New York. 
Popescu-Gorj, A. \& I. Draghia. 1974. Ord. Lepidoptera. In L'entomofaune du “grind” Saraturile-Sf.

Gheorghe (Delta du Danube). Trav. Mus. Hist. Nat. "Gr. Antipa”. Ed. Mus. d'Hist. Nat. "Gr. Antipa", Bucarest. 14: 157-173.

Popescu-Gorj, A. \& F. Konig. 1976. Ord. Lepidoptera. In Contributions a la connaissance de la faune du departement Vrancea. Trav. Mus. Hist. Nat. "fr. Antipa”. Ed. Mus. d'Hist. Nat. "Gr. Antipa”, Bucarest. 17: 303-307.

Seitz, A. 1914. The Macrolepidoptera of the world. Noctuidae III. Stuttgart, Germany.

Spüler, A. 1908. Die Schmetterlinge Europas. I. Band. E. Schweizerbartsche Verlagsbuchhandlung. Stuttgart, Germany.

Wiklund, C. 1975. The evolutionary relationship between adult oviposition preferences and larval host plant range in Papilio machao L. Oecologia (Berl.) 18: 185-197. 